University of Nebraska - Lincoln

DigitalCommons@University of Nebraska - Lincoln

July 2000

\title{
Effective surface Debye temperature for NiMnSb.100. epitaxial films
}

C.N. Borca

University of Nebraska-Lincoln

Takashi Komesu

University of Nebraska-Lincoln, tkomesu2@unl.edu

Hae-kyung Jeong

University of Nebraska-Lincoln, hjeong@unl.edu

Peter A. Dowben

University of Nebraska-Lincoln, pdowben@unl.edu

D. Ristoiu

Laboratoire L. Ne'el, CNRS, BP 166 X, 38042 Grenoble Cedex 9, France

See next page for additional authors

Follow this and additional works at: https://digitalcommons.unl.edu/physicsdowben

Part of the Physics Commons

Borca, C.N.; Komesu, Takashi; Jeong, Hae-kyung; Dowben, Peter A.; Ristoiu, D.; Hordequin, Ch.; Pierre, J.; and Nozie`res, J. P. , "Effective surface Debye temperature for NiMnSb.100. epitaxial films" (2000). Peter Dowben Publications. 29.

https://digitalcommons.unl.edu/physicsdowben/29

This Article is brought to you for free and open access by the Research Papers in Physics and Astronomy at DigitalCommons@University of Nebraska - Lincoln. It has been accepted for inclusion in Peter Dowben Publications by an authorized administrator of DigitalCommons@University of Nebraska - Lincoln. 


\section{Authors}

C.N. Borca, Takashi Komesu, Hae-kyung Jeong, Peter A. Dowben, D. Ristoiu, Ch. Hordequin, J. Pierre, and J. P. Nozie`res 


\title{
Effective surface Debye temperature for $\mathrm{NiMnSb}(100)$ epitaxial films
}

\author{
C. N. Borca, Takashi Komesu, Hae-kyung Jeong, and P. A. Dowben ${ }^{\text {a) }}$ \\ Department of Physics and Astronomy and the Center for Materials Research and Analysis, \\ Behlen Laboratory, University of Nebraska, Lincoln, Nebraska 68588-0111 \\ D. Ristoiu, Ch. Hordequin, J. Pierre, and J. P. Nozières \\ Laboratoire L. Néel, CNRS, BP 166 X, 38042 Grenoble Cedex 9, France
}

(Received 10 March 2000; accepted for publication 12 May 2000)

\begin{abstract}
The surface Debye temperature of the NiMnSb (100) epitaxial films has been obtained using low energy electron diffraction, inverse photoemission, and core-level photoemission. The normal dynamic motion of the (100) surface results in a value for the effective surface Debye temperature of $145 \pm 13 \mathrm{~K}$. This is far smaller than the bulk Debye temperature of $312 \pm 5 \mathrm{~K}$ obtained from wave vector dependent inelastic neutron scattering. The large difference between these measures of surface and bulk dynamic motion indicates a soft and compositionally different (100) surface.

(c) 2000 American Institute of Physics. [S0003-6951(00)05127-5]
\end{abstract}

Interest in NiMnSb Heusler alloys remains active because of the relatively high Curie temperature $(\sim 730 \mathrm{~K})$ and like all half metallic systems has potential technological applications in spin polarized electron transport devices. ${ }^{1}$ In spin tunnel junction devices, the interfaces play an important role in the value of the tunneling magnetoresistance (TMR). The interface should have, ideally, $100 \%$ polarization at the Fermi energy and well characterized "matched" Fermi crossings. Unfortunately, the polarization of NiMnSb is very sensitive to composition and defects ${ }^{2,3}$ and the $\mathrm{NiMnSb}(100)$ surface is observed to be very fragile. ${ }^{2,4}$ The thermal motions of the surface atoms dictates many of the physical and chemical properties of a surface, such as surface diffusion, ${ }^{5}$ anharmonicity leading to roughening transitions, ${ }^{6}$ surface reconstructions, ${ }^{7}$ and influence the extent of composition inhomogenities, including defects. The key descriptive parameter of the dynamic motions of atoms on the surface, as well as in the bulk, is the Debye temperature.

In this letter we investigate the effective surface Debye temperature of the $\mathrm{NiMnSb}(100)$ which we compare to the bulk Debye temperature calculated from the phonon dispersion curves obtained from inelastic neutron scattering. This comparison is one of only a limited number of such studies in complex compound materials ${ }^{8,9}$ and the first for the Heusler alloys.

The bulk Debye temperature of single crystals can be estimated from application of the Debye model and the phonon dispersion curves. ${ }^{10}$ In this model, the upper limit $v_{D}$ to the frequency is to be obtained from the normalizing condition that the total number of oscillatory modes is equal to $3 r N_{A}$ per mole, where $r$ is the number of atoms per unit cell (and $N_{A}$ is Avogadro's number). The quantity $v_{D}$ is with the related parameter $\theta_{D}$ by

$$
v_{D}=\frac{k_{B}}{h} \theta_{D},
$$

where $h$ is Planck's constant and $k_{B}$ is Boltzman's constant. Thus, the frequency limit ${ }_{D}$ and the related parameter $\theta_{D}$, the Debye temperature, can be obtained from the formula ${ }^{10}$

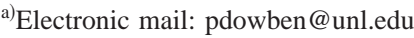

$$
\frac{4 \pi V}{3}\left(c_{L}^{-3}+2 c_{T}^{-3}\right) v_{D}^{3}=3 r N_{A},
$$

in which $V$ is the molar volume and $c_{L}, c_{T}$ are the corresponding longitudinal and transversal wave velocity. For cubic crystals, the velocities are related to the elastic constants $C_{11}, C_{44}$, and $C_{12}$ along the main axis of symmetry (100), (110), and (111), as described in Ref. 11. For values of the scattering vector $q$ close to zero, the dispersion of the acoustic oscillatory modes is linear, and the slope of this linear dependence is related to particular elastic constants, depending on the scanning direction.

The effective surface Debye temperature of single crystal materials can be investigated by low energy electron diffraction (LEED), ${ }^{12-14}$ x-ray photoemission spectroscopy (XPS), or valence band photoemission (PES) ${ }^{15}$ inverse photoemission ${ }^{16}$ as well as atom beam scattering and other surface sensitive techniques (Ref. 15 and the references therein). Generally, in electron scattering and electron spectroscopy techniques it is assumed, in the absence of a surface phase transition, that the emerging electron beam intensity depends exponentially on the sample temperature $e^{13,14}$

$$
\begin{aligned}
& I=I_{0} \exp (-2 W) \\
& 2 W=\frac{3 \hbar^{2} T(\Delta k)^{2}}{2 m k_{B} \theta_{D}^{2}},
\end{aligned}
$$

where $W$ is the Debye-Waller factor, $\hbar$ is Planck constant, $T$ is the sample temperature, $\hbar(\Delta k)$ is the electron momentum transfer, $m$ is the mass of the scattering center, $k_{B}$ is the Boltzmann constant, and $\theta_{D}$ is the effective surface Debye temperature. This surface $\theta_{D}$ is dominated by the dynamic motions normal to the surface in almost all experiments and typically does not contain significant in-plane or anharmonic contributions to the true surface Debye temperature, ${ }^{15}$ and therefore tends to be independent of surface orientation. [The major exception is high-resolution electron energy loss spectroscopy of the surface (where the details of phonon dispersion can matter) and, in off-specular scattering geometries, nondipole active modes can be probed]. 


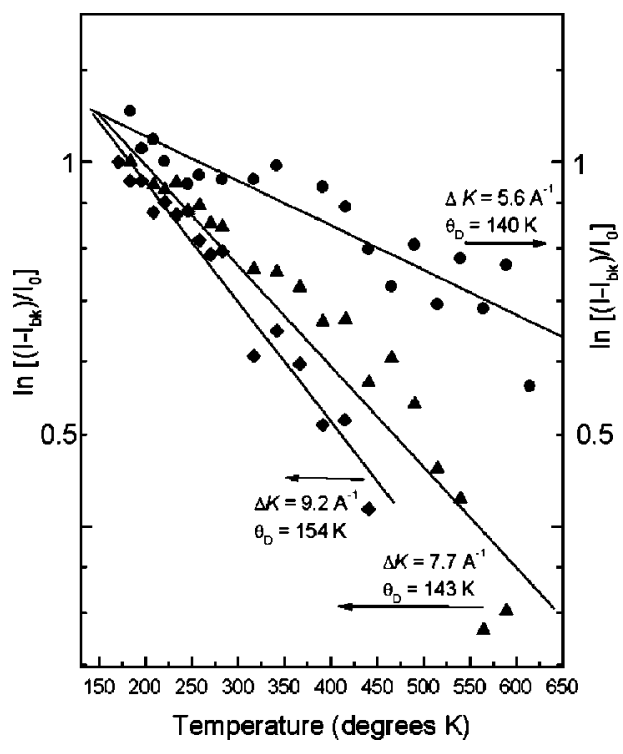

FIG. 1. Logarithm of the intensities of the diffracted electron spots obtained in LEED, after background $\left(I_{b k}\right)$ subtraction and normalization to the value $\left(I_{0}\right)$ at the lowest temperature. The three sets of data were obtained using different incident electron energies: 33 (circles), 58 (up triangle), and $83 \mathrm{eV}$ (diamond).

To apply Eqs. (3) and (4) to LEED or photoemission, we need to consider the geometry of the experiment and the mass of the scattering centers. For LEED, we have used diffracted electron beams with the scattering vector $(\Delta k)$ close to the normal to the surface (leading to the dominant role of normal lattice vibrations). The mass of the scattering centers is an average of the three different atomic masses contained in a unit cell with the scattering vector being calculated from

$$
\Delta k=2 k \cos \frac{\theta}{2}=\frac{4 \pi}{\lambda} \cos \frac{\theta}{2},
$$

where $\theta$ is the angle between the incoming and outgoing electron beams and $\lambda$ is the wavelength of the electrons.

In the case of photoemission spectroscopy (XPS and PES) $(\theta=0)$, the momentum transfer is equal to the momentum of the emitted electron: $\Delta k=2 \pi / \lambda$. In our experiments, we monitored the photoelectron intensity from the $3 d$ shell of the $\mathrm{Sb}$ atoms (using $\operatorname{Mg} K \alpha$ radiation) and appropriately corrected for the scattering center mass. For inverse photoemission, we used the momentum of the incident electron, probing the unoccupied band closest to $E_{F}(9.5-11.5 \mathrm{eV}$ electron kinetic energy). For all surface studies, the surface of the NiMnSb(100) epitaxial thin films ${ }^{17}$ of $1000 \AA$ thickness were carefully prepared by gentle Ar-sputtering and annealing treatments, as described in Ref. 2.

Figure 1 shows the temperature dependence of the intensities of the backscattered low electron diffraction spots, after background subtraction $\left(I_{b k}\right)$ and normalization to the value $\left(I_{0}\right)$ at the lowest temperature. The three sets of data shown were taken using different incident electron energies of 33,58 , and $83 \mathrm{eV}$. The angle $\theta$ from Eq. (2) varied very little in our experiment and the change in scattering vector $(\Delta k)$ largely comes from the different electron energies employed.

The change in LEED intensities, shown in Fig. 1 as the slopes of $\ln \left[\left(I-I_{b k}\right) / I_{0}\right]$, increase with increasing $(\Delta k)$ or with increasing incident (and backscattered) electron ener-
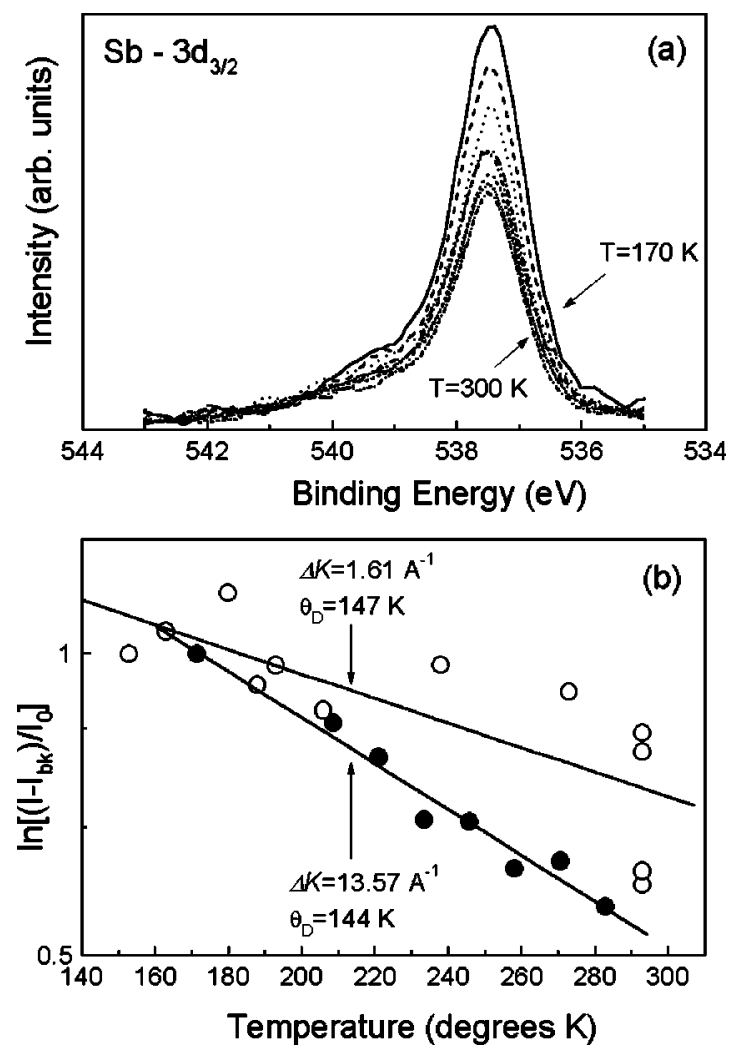

FIG. 2. (a) Normal x-ray photoemission spectra of $\mathrm{Sb}-3 d_{3 / 2}$ core level for various temperatures after background subtraction. The light was incident at $60^{\circ}$ with a photon energy of $1254 \mathrm{eV}$. (b) Logarithm of the intensity of the $\mathrm{Sb}-3 d_{3 / 2}$ core level vs temperature $(-)$ and the intensity of the state at the conduction band edge (just above $E_{F}$ ) in inverse photoemission $(\bigcirc)$.

gies. Using Eq. (1), the values obtained for the effective surface Debye temperature are $140 \pm 20 \mathrm{~K}$ (at $33 \mathrm{eV}$ incident electron energy), $143 \pm 20 \mathrm{~K}$ (at $58 \mathrm{eV}$ ), and $154 \pm 20 \mathrm{~K}$ (at $83 \mathrm{eV})$. The electron mean free path increases and so does the probing depth with increasing incident (and backscattered) electron energies. We consider the effective Debye temperature obtained at the lowest electron energies as the most representative of the dynamic normal atomic motions in the thin film surface region. The other two values, at higher electron energies, include the normal vibrations of the atoms situated increasingly deeper in the selvedge (near surface) region.

Temperature dependence of the $3 d_{3 / 2}$ core level of antimony obtained in x-ray photoemission is shown in Fig. 2. This particular core level has a narrow and intense line shape. From Eq. (4) results a value of $144 \pm 10 \mathrm{~K}$ for the effective surface Debye temperature. The mean free path of the ejected electrons in normal emission is no more than 20 $\AA$ from the free surface and so, the sample probing depth is limited to the surface and selvedge region. Inverse photoemission is also very surface sensitive. The intensity of the largely spin majority unoccupied band (near $E_{F}$ where the electron phonon coupling is greatest), ${ }^{2}$ in inverse photoemission, provides a value for the effective Debye temperature of about $147 \pm 30 \mathrm{~K}$. The value of the effective Debye temperature obtained in XPS and inverse photoemission are both representative of the harmonic vibrations of the atoms in the surface region along the film normal.

For comparison, we have compared the above values 


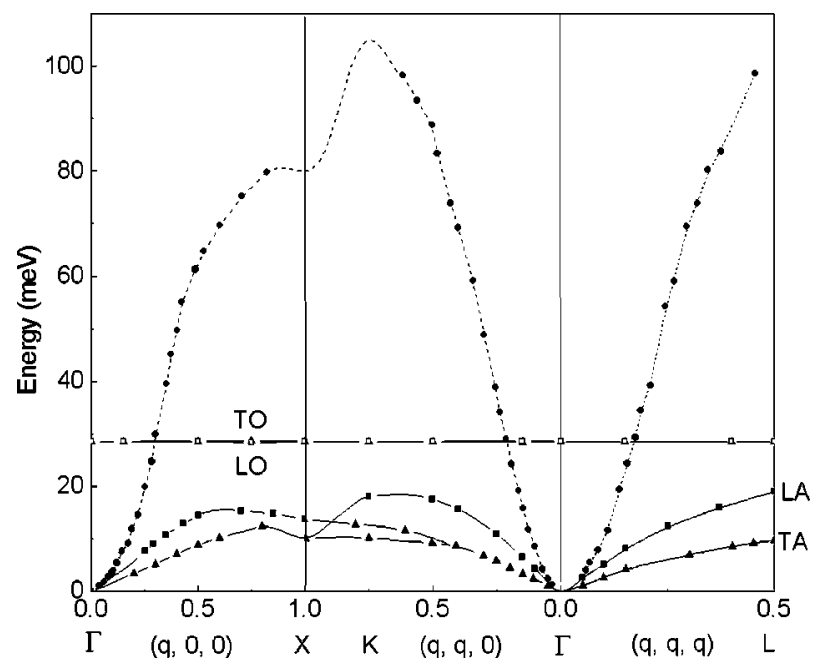

FIG. 3. Dispersion curves for magnons (-), longitudinal phonons ( $\mathbf{\square})$, and transversal phonons $(\mathbf{\Delta})$ at $300 \mathrm{~K}$. No magnetic field was applied.

with that of bulk single crystals obtained from neutron scattering (a bulk sensitive technique). Inelastic neutron scattering experiments ${ }^{19}$ were carried out at the Institute LaueLangevin, using IN1 (hot neutrons) and IN8 (thermal neutrons) spectrometers, in order to cover all the values for the transfer energies from 0 to $120 \mathrm{meV}$. Excitations were investigated mainly at room temperature in the direction of the high symmetry axis (100), (110), and (111). No magnetic field was applied. Constant energy scans were generally performed to investigate the steep magnon dispersion, whereas constant- $q$ scans were used to locate the acoustic and optical phonons. Figure 3 presents the complete experimental phonons and magnons dispersion curves at $300 \mathrm{~K} .{ }^{18}$ For a material with three atoms in the primitive cell, we generally expect three acoustic and six optical branches, whereas only one (two fold degenerated) branch of magnons was observed. Longitudinal acoustic phonons reach a maximum energy for $q$ close to $(0.6,0,0)$ in the $\langle 100\rangle$ direction and $(0.7$, $0.7,0)$ in the $\langle 110\rangle$ direction. One very flat optical branch was observed at an energy of about $29 \mathrm{meV}$, with little difference in energy for longitudinal and transverse directions. (One explanation for this dispersionless optical mode is the arrangement of the $\mathrm{Ni}$ atoms in the cubic structure. Among the closest neighbor lattice sites to nickel are the vacancy sites.)

The elastic constants can be easily extracted from the slope of the acoustic modes in each $\Gamma$ point, depending on the scanning direction. For the $\langle 100\rangle$ longitudinal mode, $C_{11}=138 \mathrm{GPa}$, for the $\langle 110\rangle$ transversal mode $1, C_{44}$ $=45 \mathrm{GPa}$ and for the $\langle 110\rangle$ transversal mode $2, C_{12}$ $=48 \mathrm{G} \mathrm{Pa} .{ }^{18}$ Using these values to calculate the longitudinal and transversal mode velocities in Eq. (2), we obtain a value of the bulk Debye temperature of $312 \pm 5 \mathrm{~K}$.

Almost independent of surface orientation we would expect that the effective surface Debye temperature is of the order of $70 \%$ (i.e., $\frac{1}{\sqrt{2}}$ ) of the bulk value of the Debye temperature. ${ }^{13-15}$ This is due to the fact that the surface atoms have only half of the number of nearest neighbors that surround the bulk atoms. ${ }^{14}$ Comparing the values of the Debye temperature obtained for the NiMnSb(100) Heusler alloy, the surface temperature is almost $45 \%$ of the bulk value. Downloaded 06 Sep 2006 to 129.93.16.206. Redistribution subject
This drop in the Debye temperature between the surface and the bulk is not unexpected if we consider that the surface and selvedge region has a different composition than the bulk, under our preparation conditions. ${ }^{2,4}$ Indeed, as shown in Ref. 4, manganese segregation occurs at the $\mathrm{NiMnSb}(100)$ surface. ${ }^{4}$ In addition, there are several other phenomena that may contribute to the lowering of the surface Debye temperature with respect to the bulk. The motion of the surface atoms is not isotropic, the force constants at the surface can be appreciably different than in the bulk and, in general, the surface may contain anharmonic phonon modes that differ from the bulk. ${ }^{20,21}$

In conclusion, we have found the effective surface Debye temperature for the $\mathrm{NiMnSb}(100)$ thin film to be 145 $\pm 13 \mathrm{~K}$, far smaller than the bulk Debye temperature of $312 \pm 5 \mathrm{~K}$. The reduced surface Debye temperature will lower the barriers to surface segregation and can be a consequence of the compositional difference due to segregation.

This work was supported by NSF through Grant No. DMR-98-02126, the Center for Materials Research and Analysis (CMRA) and the Nebraska Research Initiative at the University of Nebraska, and the Region Rhone-Alpes through the 'Nanotechnologie' program under Contract No. PR97024.

${ }^{1}$ C. Hordequin, J. P. Nozières, and J. Pierre, J. Magn. Magn. Mater. 183, 225 (1998); C. T. Tanaka, J. Novak, and J. S. Moodera, J. Appl. Phys. 81, 5515 (1997); C. T. Tanaka, J. Novak, and J. S. Moodera, J. Appl. Phys. 86, 6239 (1999)

${ }^{2}$ Delia Ristoiu, J. P. Nozières, C. N. Borca, T. Komesu, H-K. Jeong, and P. A. Dowben, Europhys. Lett. 49, 624 (2000).

${ }^{3}$ D. Orgassa, H. Fujiwara, T. C. Schulthess, and W. H. Butler, Phys. Rev. B 60, 13237 (1999)

${ }^{4}$ D. Ristoiu, J. P. Nozières, C. N. Borca, B. Borca, and P. A. Dowben, Appl. Phys. Lett. 76, 2349 (2000).

${ }^{5}$ S. C. Wang and G. Ehrlich, Surf. Sci. 206, 457 (1988); H. Roux, A. Piquet, R. Uzun, and M. Drechsler, Surf. Sci. 59, 97 (1976).

${ }^{6}$ A. D. Badorf and E. W. Plummer, J. Electron Spectrosc. Relat. Phenom. 54/55, 541 (1990); Phys. Rev. Lett. 66, 2770 (1991); G. Benedek and J. P. Toennies, Phys. Rev. B 46, 13643 (1992).

${ }^{7}$ R. T. Tung, W. R. Graham, and A. J. Melmed, Surf. Sci. 115, 576 (1982); D. A. King and G. Thomas, Surf. Sci. 91, 201 (1980).

${ }^{8}$ C. N. Borca, J. Choi, S. Adenwalla, S. Ducharme, P. A. Dowben, L. Robertson, V. M. Fridkin, S. P. Palto, and N. Petukhova, Appl. Phys. Lett. 74, 347 (1999).

${ }^{9}$ J. Zhang and E. W. Plummer, Surf. Sci. 393, 64 (1997).

${ }^{10}$ E. S. R. Gopal, in Specific Heats at Low Temperatures (Plenum, New York, 1966), p. 29.

${ }^{11}$ Charles Kittel, in Introduction to Solid State Physics (Wiley, New York, 1996), p. 90.

${ }^{12}$ E. A. Soares, V. E. deCarvalho, and V. B. Nascimento, Surf. Sci. 431, 74 (1999); S. Mroz and A. Mroz, Surf. Sci. 109, 444 (1981).

${ }^{13}$ M. A. Van Hove, W. H. Weinberg, and C.-M. Chan, in Low-Energy Electron Diffraction (Springer Series in Surface Science, New York, 1986), Vol. 6, pp. 134.

${ }^{14}$ L. J. Clarke, Surface Crystallography (Wiley, New York, 1985).

${ }^{15}$ C. Waldfried, D. N. McIlroy, J. Zhang, P. A. Dowben, G. A. Katrich, and E. W. Plummer, Surf. Sci. 363, 296 (1996).

${ }^{16}$ J. Choi, P. A. Dowben, S. Pebley, A. V. Bune, S. Ducharme, V. M. Fridkin, S. P. Palto, and N. Petukhova, Phys. Rev. Lett. 80, 1328 (1998).

${ }^{17}$ D. Ristoiu, J. P. Nozières, and L. Ranno, J. Magn. Magn. Mater. (in press).

${ }^{18}$ C. Hordequin, Ph.D. thesis (unpublished).

${ }^{19}$ C. Hordequin, J. Pierre, and R. Currat, J. Magn. Magn. Mater. 162, 75 (1996).

${ }^{20}$ L. Yang and T. S. Rahman, Phys. Rev. Lett. 67, 2327 (1991).

${ }^{21}$ P. D. Ditlevsen, P. O. Stoltze, and J. K. Nørskov, Phys. Rev. B 44, 13002 (1991). 